\title{
Sex Bias and Social Influences on Savanna Chimpanzee (Pan troglodytes verus) Nest Building Behavior
}

\author{
Fiona A. Stewart ${ }^{1} \cdot$ Jill D. Pruetz ${ }^{2}$
}

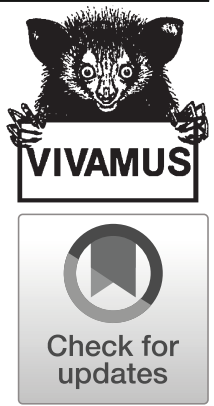

Received: 13 October 2019 / Accepted: 14 April 2020 / Published online: 18 June 2020

(C) The Author(s) 2020

\begin{abstract}
Many primates show sex differences in behavior, particularly social behavior, but also tool use for extractive foraging. All great apes learn to build a supportive structure for sleep. Whether sex differences exist in building, as in extractive foraging, is unknown, and little is known about how building skills develop and vary between individuals in the wild. We therefore aimed to describe the nesting behavior of savanna chimpanzees (Pan troglodytes verus) in Fongoli, Senegal to provide comparative data and to investigate possible sex or age differences in nest building behaviors and nest characteristics. We followed chimpanzee groups to their night nesting sites to record group (55 nights) and individual level data (17 individuals) on nest building initiation and duration (57 nests) during the dry season between October 2007 and March 2008. We returned the following morning to record nest and tree characteristics ( 71 nests built by 25 individuals). Fongoli chimpanzees nested later than reported for other great apes, but no sex differences in initiating building emerged. Observations were limited but suggest adult females and immature males to nest higher, in larger trees than adult males, and adult females to take longer to build than either adult or immature males. Smaller females and immature males may avoid predation or access thinner, malleable branches, by nesting higher than adult males. These differences suggest that sex differences described for chimpanzee tool use may extend to nest building, with females investing more time and effort in constructing a safe, warm structure for sleep than males do.
\end{abstract}

Keywords Bed building $\cdot$ Great ape $\cdot$ Sleep $\cdot$ Social learning $\cdot$ Tool use

Handling Editor: Erin G. Wessling

Electronic supplementary material The online version of this article (https://doi.org/10.1007/s10764-02000157-4) contains supplementary material, which is available to authorized users.

Fiona A. Stewart

f.a.stewart@ljmu.ac.uk

Extended author information available on the last page of the article 


\section{Introduction}

Many primates show sex differences in physical and social development, for example in social interactions such as grooming, play, and the development of complex foraging skills (reviewed in Lonsdorf 2017). Among tool-using primates, females are hypothesized to invest more in extractive foraging than males owing to the nutritional demands of pregnancy and lactation (Gumert et al. 2011; Lonsdorf 2017; McGrew 1992), although larger bodied capuchin (Cebus capucinus) males use tools more often than females, likely because of the energetic demand of using very large tools relative to body size in contrast to other species which use relatively smaller tools (Barrett et al. 2018). Juvenile male primates tend to engage in more play, and rough-and-tumble play specifically (Meredith 2015), than females and there is some evidence of greater object manipulation play in juvenile male chimpanzees (Pan troglodytes) than in females (Koops et al. 2015). In contrast, female primates show a bias toward play parenting or mothering behaviors (reviewed in Lonsdorf 2017). Such sex biases in behavior during development in primates are related to social organization and are subject to selection for the skills necessary for survival and reproduction in adulthood (Lonsdorf 2017).

Studies of intrasite variation in tool use behavior have focused on sex differences in foraging tool use, which has implications for the evolution of sexual division of labor in humans. For example, adult female chimpanzees at Gombe, Tanzania, termite fish for longer periods and more often than adult males, while males hunt more often than females (McGrew 1979), and adult females at Taï, Ivory Coast, are more efficient at nut-cracking with stone tools than are males (Boesch and Boesch 1984). Female chimpanzees in Fongoli, Senegal, account for most of the tool-assisted hunting that occurs in this dry, savanna woodland dwelling community, although males are responsible for most hunting overall (Pruetz et al. 2015). Sex differences in termite fishing skills begin early during development and suggest that males' techniques and tools have less fidelity with the mothers' than juvenile females', because males invest more time in play and social activities from a young age (Lonsdorf et al. 2004).

Although most studies of sex differences in tool use discuss tool use for extractive foraging, sex differences also occur in the manipulation of objects and use of sticks in play in wild juvenile chimpanzees (Kahlenberg and Wrangham 2010; Koops et al. 2015). A female bias has also been described in a wide range of tool-using behaviors, from self-maintenance to social play, in chimpanzees and bonobos (Pan paniscus) in captivity (Gruber et al. 2010). Sex differences in a range of behaviors including social play, motor development, and spatial independence emerge early in infancy and mirror sex-specific social roles seen in adult chimpanzees (Lonsdorf et al. 2014). Similar differences in learning and behavioral development are seen in human children (reviewed in Lonsdorf 2017; McIntyre and Edwards 2009), suggesting that some biologically based sex differences may have been present in the common ancestor of chimpanzees and humans (Lonsdorf et al. 2014).

In all hominoid species, individuals build nests just before or at sunset (Fruth 1995; Fruth and Hohmann 1993; Goodall 1962; Groves and Sabater Pi 1985). Great ape genera make broadly similar nests, despite great differences among them in habitat and social life (reviewed in Anderson et al. 2019; Fruth and Hohmann 1996). Comparisons of skill in wild-born and captive-born chimpanzees (Pan troglodytes) in captivity show that nest building is a learned behavior (Bernstein 1962; Videan 2006a). Some nest 
building behavior, or simply the disposition to build a nest, may be innate, as many captive individuals gather materials for sleep, but only individuals with appropriate experience construct full and complex nests (Bernstein 1962; Videan 2006a). The time taken for the whole group to complete nest building varies with group size, weather, and species (Fruth and Hohmann 1993; Groves and Sabater Pi 1985). Chimpanzees in Gombe, Tanzania spent on average $12 \mathrm{~h}$ per night in their nests (Lodwick et al. 2004), but few studies have observed great apes overnight in the wild (cf. Pruetz 2018; Zamma 2014). In captivity, chimpanzees sleep for only $c a .8 \mathrm{~h}$ per night, and their sleep patterns are influenced by temperature, relative humidity, age, and sex (Videan 2006b). In the wild the active period is also influenced by sex and by sexual receptivity in females, with nonreceptive females remaining in their nests longer than males or receptive females (Lodwick et al. 2004). The social dynamics of the group (e.g., dominance and mating interactions) and the trade-off with daily foraging requirements influenced the length of the active period in the wild (Lodwick et al. 2004).

Sex differences in nest building have been described in some form across all of the great apes. In all species, females nest higher than males, and orangutan, chimpanzee, and bonobo females build more day nests than males do (Brownlow et al. 2001; Fruth 1995; Fruth and Hohmann 1996). Bonobo females build nests earlier in the evening than males do (Fruth and Hohmann 1993) and silverback male gorillas (Gorilla gorilla) initiate group nest building (Groves and Sabater Pi 1985). Fruth (1995) proposed that decision-taking about nesting may be dominance related.

Savanna chimpanzees live in the most extreme environments where chimpanzees survive today (McGrew et al. 1981; Pruetz 2007; Pruetz and Bertolani 2009; Wessling et al. 2018a,b) and several differences have long been hypothesized in the behaviors of chimpanzees living in these dry, hot habitats (Moore 1996). Research on savannadwelling chimpanzees has begun to reveal the behavioral strategies these apes use to live in such habitats, including unique tool use (Hernandez-Aguilar et al. 2007; Pruetz et al. 2015; Pruetz and Bertolani 2007), differences in their social grouping and activity budget (Lindshield et al. 2017; Pruetz 2018; Pruetz and Bertolani 2009), and thermoregulatory strategies (Pruetz 2007, 2018; Pruetz and Bertolani 2009; Wessling et al. 2018a,b). Given the extreme conditions, such dry habitat chimpanzees may also adjust their timing of nesting and sleep to extend the length of the day, to accomplish the required social and foraging activities. This hypothesis is supported by observations of extensive nocturnal activity at Fongoli in three peaks overnight (immediately after nightfall, in the middle of the night, and pre-dawn), which is influenced by factors including fuller moon phases, dry season, and the presence of females with tumescent sexual swellings (Pruetz 2018). The apes socialized, fed, and moved at night and were hypothesized to be compensating for high heat stress conditions that make daytime feeding challenging (Pruetz 2018). The first peak in nocturnal activity likely occurs before Fongoli chimpanzees build their night nests (Pruetz 2018), thereby extending their day and resulting in later nesting times.

No quantitative cross-species comparisons of nest architecture or detailed longitudinal studies of the ontogeny of nest building are yet available (Anderson et al. 2019). However, the first attempts at nest building occur during the day at 8-12 mo of age but individuals do not begin to make independent night nests until after weaning at 3-5 yr of age (Van Lawick-Goodall 1968). The development of this skill resembles that of other technological skills; e.g., termite fishing is mastered between 2.5 and $5.5 \mathrm{yr}$ 
(Lonsdorf 2005), and nut-cracking between 3 and 7 yr (Biro et al. 2006). In Mahale, Tanzania, young females spend more time ant fishing and nest making than males at all ages (Hiraiwa-Hasegawa 1989).

We studied nest building during the dry season in chimpanzees at Fongoli. The aims of this study are twofold. First, we aim to describe the nesting of these savanna chimpanzees and to provide comparative data on nesting times, duration of building, and activity in nests and nest groups. We hypothesize that nest building time, duration, and initiation vary across the dry season in Fongoli as conditions become increasingly dry and hot in this savanna habitat. Specifically, we predict that

1) The onset of night nesting will be later in Fongoli compared to what is known for other great apes living in tropical forest.

2) The onset of building occurs later as the dry season progresses, as chimpanzees may extend their foraging time as hotter and drier conditions increase.

3) The duration of group building increases with group size, and that this relationship is greater than a simple linear relationship, as some individuals may continue foraging during the evening after group nesting is initiated.

4) Males initiate nest building more frequently than females, which may extend their foraging time given higher energetic demands of pregnancy and lactation.

Second, we aim to investigate sex and age differences in nest building behaviors, activities in the nest, and in nest and tree characteristics during the extreme conditions of the dry season. We hypothesize that the sex differences described for chimpanzee tool use extend to nest building, with females investing more time and effort in constructing a safe, warm structure for sleep and juveniles of each sex building nests in ways that resemble adults of that sex. Such differences may be exaggerated by the extreme weather conditions; for example, the dry season in Fongoli is initially characterized by very cold nighttime temperatures, and later by seasonally high winds (Stewart et al. 2018). There was only one immature female in the Fongoli community at the time of this study and observations of immature male nestbuilding behavior were limited, so we could not make comprehensive comparisons across all sex/age classes. We predicted that

1) Adult females spend longer building their nests than males do.

2) Adult females and immatures build nests in "safer" locations than adult males, i.e., higher, in larger trees, and positioned higher and peripherally in tree crowns (sensu Stewart and Pruetz 2013).

\section{Methods}

We collected data at Fongoli $\left(12^{\circ} 13.90 \mathrm{~N} 12^{\circ} 11.30 \mathrm{~W}\right)$ in southeastern Senegal during the dry season between October 2007 and March 2008, when there was almost no rainfall (12.2 $\mathrm{mm}$ in October and $4.7 \mathrm{~mm}$ in November), and absolute temperatures ranged $7-45^{\circ} \mathrm{C}$ (Stewart 2011). The vegetation is a mosaic woodland-savanna habitat dominated by woodland and wooded grassland, interspersed with areas of bamboo, plateau, and thicket, plus small patches of gallery forest (including "ecotone") that make up ca. 3\% of the landscape (Pruetz 2006). This region is one of the hottest, driest, 
and most open habitats for chimpanzees, with maximum daily temperature ranging $27-$ $48^{\circ} \mathrm{C}$ and averaging $37.7^{\circ} \mathrm{C}$ (Wessling et al. 2018b). Annual rainfall usually averages $<1000 \mathrm{~mm}$ (Pruetz 2018).

The Fongoli chimpanzees were fully habituated in 2005 , but only males are followed as focal individuals, to protect females from possible stress during pregnancy and lactation or as targets for poaching of infants for the pet trade (Pruetz et al. 2015). We estimated adults to be $>15 \mathrm{yr}$, adolescents 7-15 yr, juveniles 4-7 yr, and infants $<4$ yr of age (following Pruetz et al. 2015). During the study period, the community numbered 33-34 individuals, following the birth of an infant female and the death of an old male, and comprised 12 adult, 5 adolescent, 2 juvenile, and 1 infant male and 8 adult, 1 adolescent, 2 juvenile, and 3 infant females. For most of the study period, 27 individuals were weaned and building night nests independently.

\section{Nesting Behavior}

We followed focal individuals to their night nesting site to record nest building behavior and nest site location to return to the following morning for nest and tree characteristics measurements. Observations often were limited, as we could not identify individuals after nightfall, and on some occasions, we lost the party or individuals before they built their nests as tracking is more difficult after nightfall. Except for a few daytime and overnight follows, we made observations only during late afternoon and evening, until after the last individual in a party had nested. We defined night nests as nests in which chimpanzees spent the night and day nests as nests built for temporary use during the day. We recorded nests built in the evening but not slept in overnight as abandoned. If we did not observe chimpanzees leaving the nest site, we recorded it as abandoned if when we arrived at the nest site the following morning, the chimpanzees were absent and no nests in the group had fresh feces or urine beneath. We followed focal individuals until the group initiated nesting and then switched to record the first individual in sight to begin building, as it was difficult to record behavior once night had fallen. We recorded group and individual nesting behavior using a voice and/or video recorder (Memory Stick ICDMS1 Recorder) (Table I).

\section{Nest and Tree Characteristics}

FS returned the next morning to measure nest and tree characteristics. We used a clinometer (Suunto PM-5/360PC, range $0^{\circ} \pm 90^{\circ}$ and $0 \% \pm 150$, precision $1^{\circ}$ and $1 \%$ ) for all height measurements, and a tape measure for all horizontal distances. We recorded all nest and tree measurements to the nearest meter. Nest characteristics recorded included nest height, number of decisions (defined as the number of possible choices of nest location made if approaching the tree from the trunk base, e.g., a ground nest would be zero, and there are six decisions in Fig. 1; Stewart 2011), nest position within the tree crown (nest position = nest height above lowest branch height/crown height; Fig. 1), and the proportional distance of the nest from the trunk (proportion nest to trunk base $=$ nest to trunk base/crown radius; Fig. 1; Stewart 2011; Stewart and Pruetz 2013) in order to control for selected tree size. We recorded data from primary trees only (defined as trees contributing most branches to the nest structure) and included tree height and diameter at breast height (DBH, in cm; Fig. 1). 
Table I Variables recorded during observations of group and individual nest-building by chimpanzees in Fongoli, Senegal, October 2007-March 2008

\begin{tabular}{|c|c|}
\hline Variable & Data collected \\
\hline \multicolumn{2}{|c|}{ Group nest-building observations } \\
\hline Group composition & $\begin{array}{l}\text { We recorded all individuals, including number of adult/subadult, adolescent/juvenile males and } \\
\text { females, in sight during } 15 \text {-min scans. We took the last scan before nesting as the nest group } \\
\text { composition. }\end{array}$ \\
\hline Group size & Total number of nest-building individuals in the party \\
\hline Nest-building onset & Time at the start of the first nest's construction \\
\hline Nest-building duration & $\begin{array}{l}\text { Time in seconds from the start of the first nest's construction to the completion of the last nest, } \\
\text { by all individuals in the partyAfter nightfall, we recorded duration from the beginning to the } \\
\text { end of all nest-building sounds. }\end{array}$ \\
\hline Initiator & Age, sex, and identity of the first individual to build \\
\hline Last builder & Age, sex, and identity of the last builder \\
\hline Activities & $\begin{array}{l}\text { We recorded activities of individuals in the party ad libitum as feed, rest, groom, play, or travel. } \\
\text { We used these observations to determine individual builder activity. }\end{array}$ \\
\hline \multicolumn{2}{|c|}{ Individual nest-building observations } \\
\hline Builder identity & Age, sex, or individual identity \\
\hline Builder activities & Activity immediately before and after building as feed, rest, groom, play, or travel \\
\hline Start time & Time the individual manipulated first branch \\
\hline Building duration & $\begin{array}{l}\text { Time in seconds from the first branch manipulated to the last manipulation in building the nest. } \\
\text { We deducted pauses }>1 \text { min from the duration. }\end{array}$ \\
\hline Duration of use & Where nests were abandoned we noted the duration of use. \\
\hline Rest position & Supine, right side, left side, or prone \\
\hline
\end{tabular}

\section{Comparison of Age and Sex Classes}

We observed each individual building a night nest from one to seven times and sample sizes for nesting behavior, nest, and nest tree data varied (Table II; Electronic Supplementary Material Table SI). Given the small sample size of each age/sex class, we grouped adolescent and juvenile age classes as immature, which limited our ability to infer the ontogeny of nest building by comparing across age classes. Nelly was the only immature independently night nest building female in the community, and we measured her nest only once, so we did not include these data in analyses.

We analyzed group nest building behavior using multiple linear regression to disentangle the effects of time of year, sunset, and group size on the onset of nesting time (model 1) and time of year, time of onset of nesting after sunset, and group size on the duration of group nest building (model 2). We assessed normality and homogeneity of the residuals of all models by examining qq plots and plots of residuals and standardized residuals against fitted values, and standardized residuals against leverage. We assessed collinearity of models including more than one test predictor by examining a pairwise correlation 


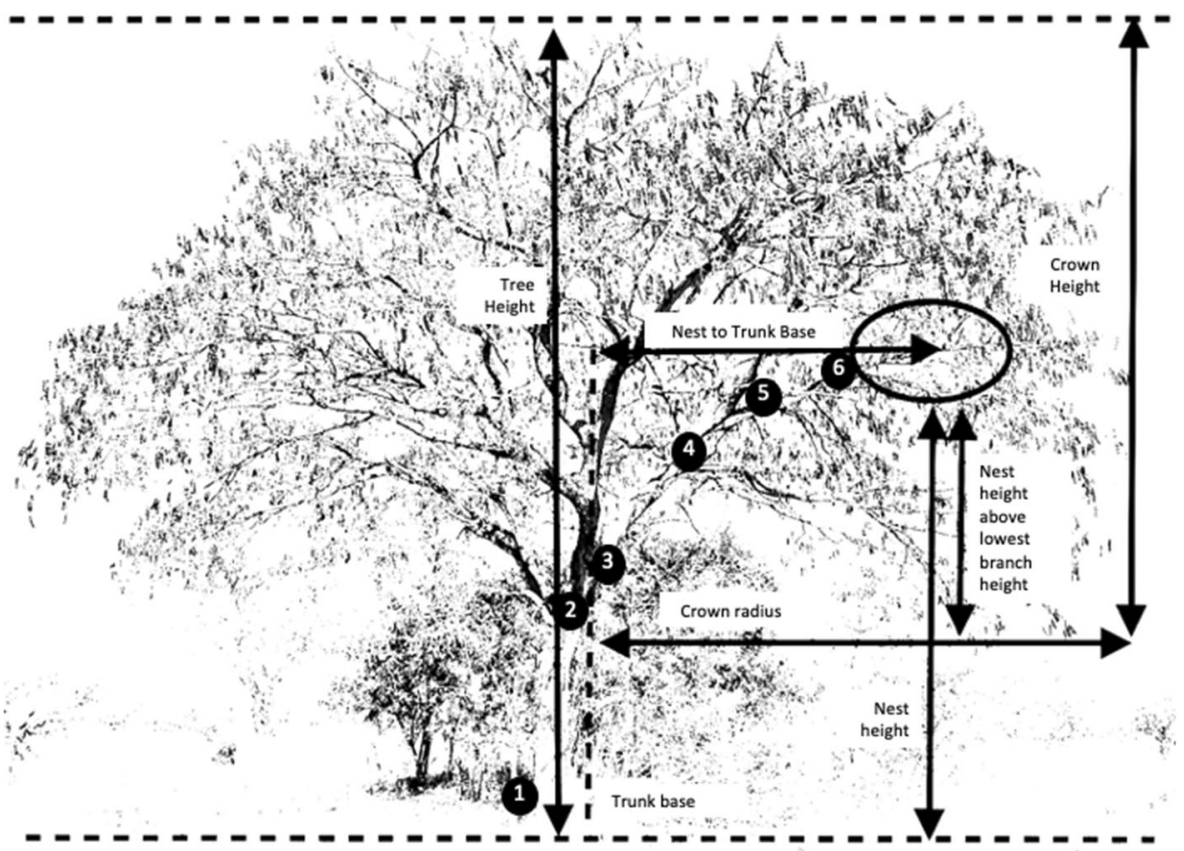

Fig. 1 Depiction of chimpanzee nest and tree characteristics measurements taken in Fongoli, Senegal, between October 2007 and March 2008. Black circles with numbers indicate each "decision" from selecting to climb the tree, through each branch fork, to the nest location (six decisions in this case), assuming that the chimpanzee approached the nest location from the ground.

matrix of variables, variance inflation factors, and tolerance. Time of year and sunset were collinear, given that sunset occurred later as the dry season progressed, so we removed time of year from these models. We obtained an overall $P$-value for all models by testing the full model against a null model without the effect in question. We identified the significance of each test predictor using a chi-square test to compare a reduced model lacking that term with a full model.

Table II Number of chimpanzee night nests recorded by sex and age in Fongoli, Senegal, October 2007March 2008

\begin{tabular}{llllll}
\hline & & \multicolumn{2}{l}{ Behavior } & & Nest/tree characteristics \\
\hline Age & Sex & Nests & Individuals & Nests & Individuals \\
Adult & Male & 43 & 10 & 37 & 10 \\
& Female & 10 & 5 & 19 & 8 \\
\multirow{2}{*}{ Immature } & Male & 4 & 2 & 14 & 6 \\
& Female & & & 1 & 1 \\
All & & 57 & 17 & 71 & 25 \\
\hline
\end{tabular}


To test whether one sex was more likely to initiate group nest building than another, we determined the probability that a female initiated nesting for a given proportion of females in the nesting group. To do this, we ran a binomial generalized linear mixed model (model 3 ) with initiator sex as the response $($ female $=1$; male $=0$ ) and the proportion of males in the group as the predictor variable. We also included group size as a covariate as a control. We tested whether the slope estimate for the effect of the proportion of males in the group was different from 1 by calculating the confidence interval (CI) of the effect size. A slope of 1 would indicate that as the number of males in the group increased, the probability of the initiator being male also increased in a linear manner. A significant deviation from this relationship (i.e., the CI did not include 1) would indicate that one sex was initiating more than expected for the proportion of that sex in the group.

We performed all analyses in R (Version 3.6.0, R Core Team 2019) and used lme4 (Bates et al. 2015) to build linear mixed models to investigate individual nest building behavior and test for sex differences in nest building behavior, and nest and nest tree characteristics. We included nest builder identity as a random effect in all models to control for repeated measures. We first investigated the relationship between nest building duration and nest type (i.e., freshly built nest used overnight, abandoned night nest, or reused night nest) by including nest type as a fixed effect into the model (model 4). Second, we tested the relationship between nest building duration and sex by including sex as a fixed effect in the model (model 5). Finally, we tested the relationship between time of onset of building and sex (model 6). We did not include date built as a random effect in models 4-6 because we observed only approximately one individual per date. Lastly, we built several models (models 7-12) to investigate the relationship between each of the recorded nest and tree characteristics (see earlier) and age/sex classes as a fixed effect. In addition to builder identity, we also included the date the nest was built as a random effect. Visual inspection of residual plots as described earlier did not reveal any obvious deviations from normality or heterogeneity. We determined an overall $P$-value for each model, but because package lme4 does not report $P$-values for within variable comparisons and given between 53 and 67 degrees of freedom in each analysis, we considered sex/age class differences to be significant at a critical value of 0.05 for a two-tailed $t$-test if the $t$-value was $>|2.00|$. We report trends with a critical value of 0.10 if the $t$-value was $>|1.67|$. Owing to limited sample sizes we report significant results here as preliminary trends that require further study.

\section{Ethical Note}

All data collected on wild chimpanzees for this article were noninvasive behavioral observations and we maintained a distance of $15 \mathrm{~m}$ to minimize potential stress to the animals and the risk of disease transmission. All research was conducted with permission and in accordance with the guidelines of the Department of Forestry in the Republic of Senegal and the IACUC guidelines of Iowa State University. The authors declare that they have no conflict of interest.

Data Availability The data sets analyzed in the current study are available from the corresponding author on reasonable request. 


\section{Results}

\section{Group Nest Building Behavior}

We observed complete group nest building on 55 nights. On four occasions we lost the chimpanzees before beginning nest building (at 19:20, 19:55, 19:45, and 20:07 h) and on two occasions FS left the group before nesting was finished because of the late time of night and distance from camp, as the chimpanzees had not finished or started nesting by $22: 30$ and 20:30 h respectively. On 5 nights when we observed complete group nestbuilding, the chimpanzees then abandoned their nests, either while observers were present or at some time later during the night.

Of 49 nest groups for which we made a complete party count, 41 were of mixed sex composition (mean group size $=9 \pm \mathrm{SD} 5.9$ individuals; mean males/group $=5 \pm \mathrm{SD}$ 2.5, $N=49$ groups; mean females/group $=2 \pm \mathrm{SD} 1.2, N=41$; mean infants/group $=2$ $\pm \mathrm{SD} 1.1, N=30$; mean immatures/group $=2 \pm \mathrm{SD} 1.3, N=30$ ).

\section{Time of Onset of Group Nest Building}

Mean time of onset of chimpanzee nest building was 18:48 \pm SD 00:48 h, while mean time of completion of group nest building was 19:17 \pm SD 00:52 h (Fig. 2). Sunset during the study ranged from 18:20 to $19: 01 \mathrm{~h}$ from the beginning to the end of the study, and mean time of beginning nest building was $27 \pm 44$ min after sunset. Group size and sunset explained $25 \%$ of the variance in time of onset of nest building (model 1: $\left.R^{2}=0.25, F_{(2,46)}=7.5, P=0.001\right)$, with larger groups nesting significantly later $(\beta=$ $0.28, t=2.1, P=0.036)$ even as groups nested later as the sun set later $(\beta=0.32, t=$ $2.4, P=0.016)$.

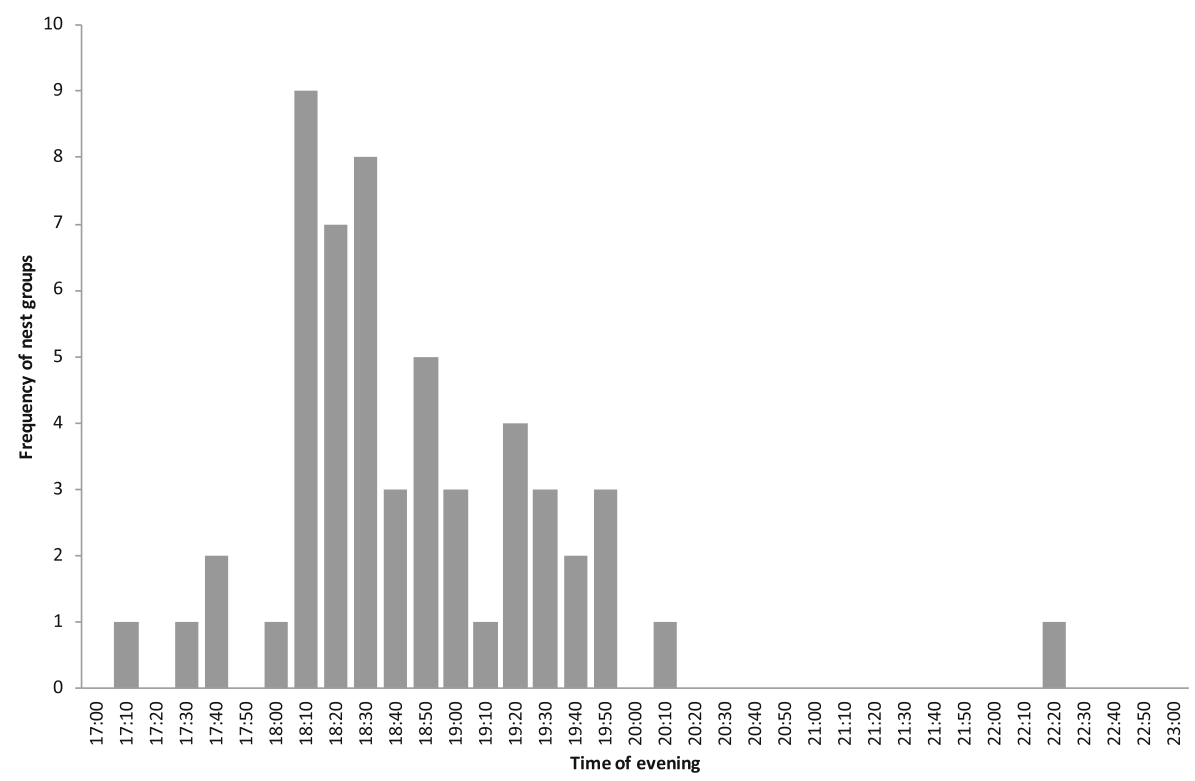

Fig. 2 Time of onset of nesting for chimpanzee nest groups in Fongoli, Senegal, at intervals of $10 \mathrm{~min}$, October 2007-March 2008. 


\section{Duration of Group Nest Building}

The mean duration of nest group construction was $29 \pm$ SD 20.4 min. Group size and time of onset after sunset explained $48 \%$ of the variance in the duration of group nest construction (model 2: $R^{2}=0.48, F_{(2,46)}=21.14, P<0.001$ ). Larger groups took significantly longer to build $(\beta=0.73, t=6.5, P<0.001)$, but built more synchronously if building started later after sunset $(\beta=-0.24, t=-2.1, P=0.039)$.

\section{Initiator of Group Nest Building}

Eighteen individuals ( 6 females and 12 males) initiated nest building during the study on one to four occasions. We found no significant deviation from a linear relationship between the proportion of males in a group and the probability of a male initiating nest building (model 3: slope $=-2.19, z=-0.94, \mathrm{df}=25, P=0.35 ; \mathrm{CI}=-6.7-2.4$ ). Females tended to initiate building in larger nest groups, although this was not significant (slope $=0.16, z=1.7, P=0.08)$.

\section{Individual Nest Building Behavior}

FS saw 60 nests built by known individuals during the study. Two of these were day nests, 5 were reused, and 14 were abandoned shortly after construction. Nests built for overnight use were built in mean $234 \pm$ SD $101 \mathrm{~s}$ (Fig. 3). Abandoned nests were used for a mean of $140 \pm$ SD $209 \mathrm{~s}$ and were built in mean $180 \pm$ SD $41 \mathrm{~s}$ (Fig. 3). Addition of fresh material when chimpanzees reused a nest took only mean $75 \pm \mathrm{SD} 41 \mathrm{~s}$. The

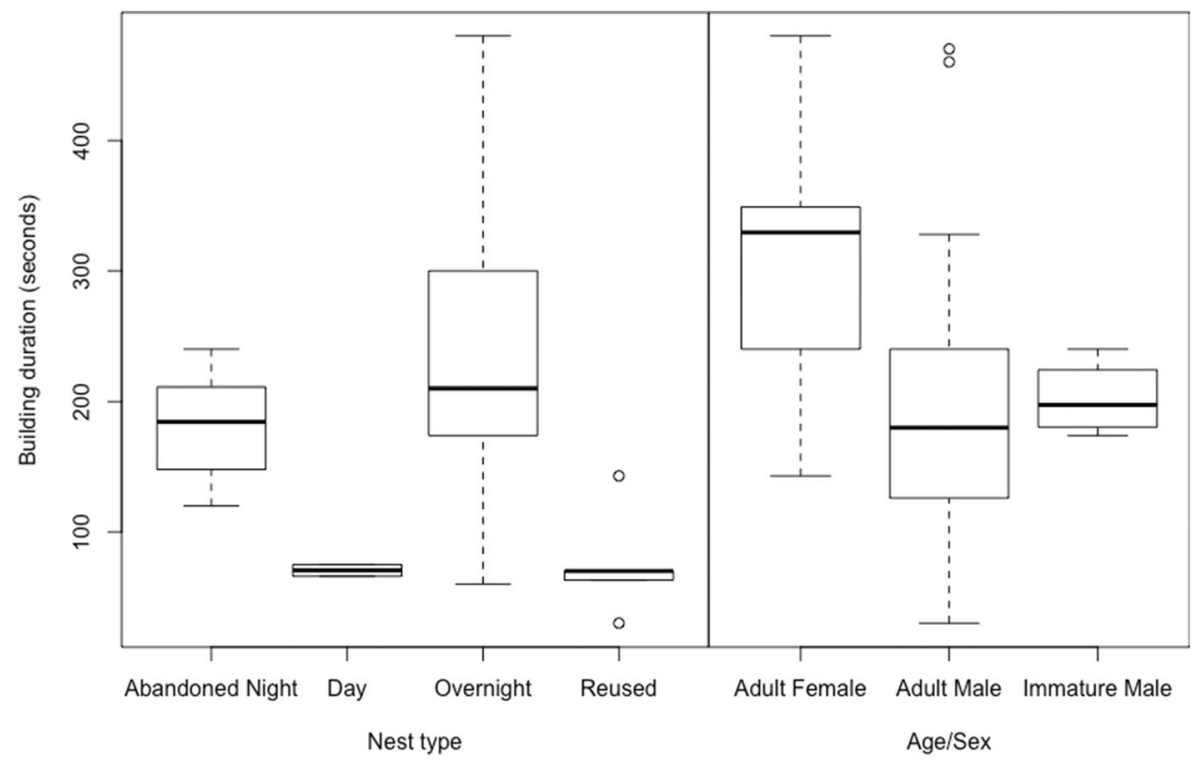

Fig. 3 Duration of building in seconds of overnight, abandoned, day, and reused chimpanzee nests, and of adult males, females, and juvenile male chimpanzees, at Fongoli, Senegal, October 2007-March 2008. Boxes indicate first and third quartile of range and the horizontal bar indicates the median. Bars outside boxes indicate range, excluding outliers, which are indicated by circles. 
two day-nests were built in less time (66 and $75 \mathrm{~s}$ ) and we excluded them from analyses. Using a linear mixed effects model (model 4), we found that building duration was significantly affected by nest type $\left(\chi^{2}=11.19\right.$, df $=2, P=0.004 ; N=$ 57, ind. = 17). Specifically, reused nests were built in significantly less time than freshly built night nests ( $\beta \pm \mathrm{SE}=-146 \pm 44, t=-3.34$ ), but the building duration of abandoned night nests was not significantly different $(\beta \pm \mathrm{SE}=-44 \pm 35, t=-1.27)$ from freshly built night nests (intercept $\beta \pm \mathrm{SE}=238 \pm 17, t=13.99$ ).

The most frequent activity in night nests immediately following construction was rest $(89 \%, N=38)$. We observed feeding thrice in night nests $(6 \%)$ and play once between a mother and infant. The behavior immediately preceding nest building was equally often feed or travel (each $49 \%, N=45$ ). Often the chimpanzees travelled several hundred meters from their last feeding tree before nesting. During rest, chimpanzees adopted a supine posture in almost all cases observed $(90 \%, N=39)$. We observed prone posture and lying on the right side only twice each.

\section{Differences in Nest Building Behavior Between Sex and Age Classes}

Using a linear mixed effects model (model 5), we found that males and females did not differ in the start time of nest building $\left(\chi^{2}=0.05\right.$, df $\left.=1, P=0.83\right)$, but did differ significantly in nest building duration (model 6: $\chi^{2}=11.37 \mathrm{df}=1, P<0.001 ; N=57$, ind. = 17). Specifically, males $(\beta \pm \mathrm{SE}=-127 \pm 34, t=-3.77)$ had shorter building durations than adult females (intercept $\beta \pm \mathrm{SE}=319 \pm 34, t=10.53$ ). We did not compare building duration of age classes statistically because of the limited sample size of only four nests from two immature males, but the duration of these nests was similar to that of adult males (Fig. 3).

\section{Differences in Nest and Tree Characteristics Between Sex and Age Classes}

We measured nest and nesting tree characteristics of 70 nests of known builder identity (excluding the juvenile female), built by 24 different individuals across 29 nights (Table II). Using a linear mixed effects models, we found that age/sex classes significantly differed in nest height (model 7: $\chi^{2}=10.67$, df $=2, P=0.005$ ), number of "decisions" to nest position (model 8: $\chi^{2}=11.43 \mathrm{df}=2, P=0.003$ ), and the tree height (model 9: $\chi^{2}=6.07, \mathrm{df}=2, P=0.05$ ) and DBH of the main nesting tree (model 10: $\chi^{2}$ $=10.58, \mathrm{df}=2, P=0.005$ ), but not other measures of nest characteristics (model 11, position in tree crown: $\chi^{2}=0.86, \mathrm{df}=2, P=0.65$; model 12 , proportion Nest to Trunk Base: $\chi^{2}=0.81$, df $=2, P=0.66$ ). Specifically, adult females and immature males nested higher, with more "decisions" from the ground to the nest, and in taller trees of larger DBH, than adult males (Table III), although the difference in the height of trees selected was not significant (Table III).

\section{Discussion}

Chimpanzees in this study nested later than other communities of great apes where such data are available (median start time bonobo nesting: 17:45 h, Fruth 1995; 
Table III Summary of model results (models 7-12) testing the relationship between chimpanzee nest and nest tree characteristics and the sex and age class of the builder (excluding immature female) in Fongoli, Senegal, October 2007-March 2008

\begin{tabular}{|c|c|c|c|c|c|c|}
\hline Model & Model terms & $N$ observations & $N$ individuals & $\beta$ & SE & $t$ \\
\hline \multirow[t]{3}{*}{ Nest height } & Intercept & 37 & 10 & 4.5 & 0.71 & \\
\hline & Adult female & 19 & 8 & 3.3 & 1.05 & $3.15^{* *}$ \\
\hline & Immature male & 14 & 6 & 2.7 & 1.11 & $2.43^{*}$ \\
\hline \multirow[t]{3}{*}{ Number of decisions } & Intercept & 37 & 10 & 3.6 & 0.65 & \\
\hline & Adult female & 19 & 8 & 2.3 & 0.92 & $3.56^{* * * *}$ \\
\hline & Immature male & 13 & 6 & 2.2 & 0.98 & $2.21 *$ \\
\hline \multirow[t]{3}{*}{ Position in tree crown } & Intercept & 32 & 10 & 0.46 & 0.06 & \\
\hline & Adult female & 18 & 8 & 0.07 & 0.08 & 0.81 \\
\hline & Immature male & 12 & 5 & -0.04 & 0.10 & 0.00 \\
\hline \multirow[t]{3}{*}{ Proportion nest to trunk base } & Intercept & 28 & 10 & 0.48 & 0.08 & \\
\hline & Adult female & 16 & 8 & 0.07 & 0.13 & 0.63 \\
\hline & Immature male & 9 & 5 & 0.11 & 0.13 & 0.83 \\
\hline \multirow[t]{3}{*}{ Tree height } & Intercept & 33 & 10 & 8.8 & 1.21 & \\
\hline & Adult female & 19 & 8 & 3.4 & 1.75 & $1.95^{\wedge}$ \\
\hline & Immature male & 13 & 6 & 4.0 & 1.86 & $2.16^{*}$ \\
\hline \multirow[t]{3}{*}{ Diameter at breast height } & Intercept & 33 & 10 & 20.9 & 3.09 & \\
\hline & Adult female & 18 & 8 & 7.6 & 3.00 & $2.53 *$ \\
\hline & Immature male & 11 & 5 & 9.6 & 3.21 & $3.00 * *$ \\
\hline
\end{tabular}

${ }^{\wedge} P<0.10 ; * P<0.05 ; * * P<0.01 ; * * * P<0.001$.

start time range: 18:00-19:00 h, usually before sunset, Goodall 1962; start time range: 18:05-18:48 h, Groves and Sabater Pi 1985). That nesting occurs later as the dry season progresses in Fongoli, and chimpanzees nest more synchronously in large groups when nesting begins later, supports suggestions made in previous studies of this community showing that high temperatures may drive chimpanzees to adjust their activity toward evening and overnight (Pruetz 2018). We collected only in the dry season, and further study across all seasons in Fongoli is needed to determine whether the trends we describe here can be generalized across seasons.

We found no sex differences in which individuals initiated nest building, but several sex differences in nest building duration, and nest and tree characteristics that supported our hypotheses. Sample sizes in this study were limited owing to the difficulty of identifying nest builders in falling light conditions and because we could record female nest building only opportunistically in mixed-sex groups as we did not follow females as focal individuals (Pruetz et al. 2015). It is possible that we would find further similarities or differences in nest building behavior between the sexes if we also followed females in the absence of males. Our results are therefore preliminary but indicate that in the dry season and in the presence of males, female chimpanzees at Fongoli spend longer building nests than males do. This difference may indicate that females put more effort into nest building, although they may also be less efficient, or slower, at manipulating branches. Detailed architectural analyses of how each sex 
builds nests are necessary to clarify whether females take longer to build similar nests, or whether their nests are more complex than those of males. Limited preliminary nest architectural data from a few females suggests that females use more material, specifically building thicker nests, with more lining, larger branches, and more support branches (Stewart 2011). However, more nest architectural data are needed from a larger sample of individuals to ensure that these patterns do not simply represent idiosyncratic variation (Stewart 2011).

Selection of sleeping sites situated in taller trees, higher in trees, and more peripherally within the tree crown is well established as an antipredation strategy across primate species (Albert et al. 2011; Anderson 2000; Hamilton 1982; Smith et al. 2018, Thiry et al. 2016). We hypothesized that females and immature males nest in locations within trees thought to be more peripheral and inaccessible to predators (Stewart and Pruetz 2013) given their smaller body size. Although they nested higher in trees, females did not nest higher or more peripherally in the tree crown, but instead selected larger trees in which to nest. That females and immature males nested in locations with more "decisions" may reflect the higher height of the nests, given that there was no difference in how peripheral the nests were in the tree crown. These relationships remain to be tested across a greater range of environmental conditions in Fongoli, and with a more robust sample including more individuals and more nests per individual (Table II), but females and juveniles of other primate species are also known to select higher, peripheral sleeping sites as an antipredatory strategy (Di Bitetti et al. 2000; Reichard 1998). Further study across populations is also needed to determine whether the differences identified here are specific to this savanna woodland dwelling population. More data are also needed across all sex and age classes and longitudinally as nest building develops to better understand sex differences and how nest building skill is learned and develops. However, the variations between immature and adult males and females described here suggest that sex differences described for chimpanzee tool use and other behaviors (Lonsdorf 2005; Lonsdorf et al. 2004, 2014) extend to nest building if females' greater investment of time and effort results in a safe, warm structure for sleep.

\section{Nesting Behavior}

Few studies have reported the time of nesting of chimpanzees (cf. Goodall 1962; Groves and Sabater Pi 1985). Nesting time of chimpanzees observed for five nights in Mahale ranged between 18:47 and 19:51 h (Zamma 2014). Chimpanzees nest earlier in the wet season in Gombe, but usually do so between 18:00 and 19:15 h (Goodall 1962). Chimpanzees and gorillas in Equatorial Guinea nested between 18:00 and 18:50 $\mathrm{h}$ and 17:30 and 18:35 h respectively, and nesting time was influenced by group size, composition, and weather (Groves and Sabater Pi 1985). Bonobos in Lui Kotal, Congo, nest earlier on wetter days than on dry days and earlier than recorded here for chimpanzees, with a median start time of 17:44 h, and no groups began after 18:00 $\mathrm{h}$ when the sun set (Fruth 1995). Chimpanzees in this study nested on average after sunset, although sunset occurs later in Fongoli than in Lui Kotal, DRC (Fruth 1995), but we did not collect data during the wet season when they may nest earlier, as in other studies. Other primate species also tend to enter their sleeping sites prior to dusk, making nesting after sunset by Fongoli chimpanzees an unusual behavior across 
primates (e.g., Geoffrey's spider monkey, Ateles geoffroyi: Chapman 1989; tufted capuchin, Sapajus [Cebus] apella: Di Bitetti et al. 2000; Lar gibbon, Hylobates lar: Reichard 1998). Later nesting times and nocturnal activity may be an adaptation to extreme conditions in savanna chimpanzee habitats (Pruetz 2018), but nocturnal activity is widespread across all habitats and is influenced by high daily temperatures and low levels of human activity (Tagg et al. 2018). Chimpanzees in Kibale, Uganda have even been reported to forage on crops more during the night than during the day to avoid humans (Krief et al. 2014). Where chimpanzees position their nests and how early they rise in the morning involves flexible planning depending on the quality and ephemerality of their targeted breakfast foods (Janmaat et al. 2014). Chimpanzees may therefore be able to forward plan and be flexible in their nesting times and nocturnal activity, allowing them to respond to changing environmental factors.

In Gombe, chimpanzee males, and females with tumescent sexual swellings, have longer active periods (and so later nesting times) than nonreceptive females (Lodwick et al. 2004), while chimpanzee parties in Fongoli were significantly more active overnight when swollen females were present than when they were not (Pruetz 2018). Our data collection was biased toward nest groups containing males and a single female, Tumbo, whose sexual swelling was frequently tumescent during the study. The heightened social activities resulting from the presence of a swollen female may have influenced the late nesting times found here. Alternatively, Fongoli chimpanzee activity patterns may have been influenced by the extreme temperatures in the latter dry season, which discourage sufficient foraging or mating during the heat of the day and result in increased activity during the night (Pruetz 2018).

The duration of group nest building is similar in chimpanzees and gorillas (Groves and Sabater Pi 1985) and bonobos (Fruth 1995). Our findings are similar to those for bonobos in Lui Kotal, who take 4-90 min for the group to complete nesting and the group nests more synchronously if nesting is initiated later, which the author hypothesized allows "extra" time for foraging or travelling before nesting, compared to cold days when energy may be conserved by nesting earlier (Fruth 1995). The most frequently observed activity before night nesting in our study was travel as individuals nesting after sunset often traveled from the feeding tree together to a separate site for nest building, whereas when nesting was initiated before sunset individuals often left a feeding tree at intervals and nested close by (F. A. Stewart pers. obs.).

We found no relationship between sex and initiation of nest construction, and there may be several influences on timing of onset of nest building, as described earlier. Although dominance may play a role (Fruth 1995), females also have higher energy demands through pregnancy and lactation (Emery Thompson et al. 2012) that could also lead to females continuing to forage once males have begun building. However, more data are needed across seasons, sites, and species for a detailed comparison with regard to female receptivity and group composition and initiator of nest building.

Reuse of nests by chimpanzees is usually rare (Plumptre and Reynolds 1997), although in another savanna woodland site they reuse nests more frequently and reuse specific locations within trees, perhaps because these locations become prefabricated for future nest building (Stewart et al. 2011). Chimpanzees in the savanna mosaic of Comoé understand the appropriate reuse periods for resources other than nests, specifically Ceiba pentandra trees from which they peel bark (LaPuente et al. 2020). There is some indication that chimpanzees reuse nests at greater frequency during the dry 
season, perhaps due to a paucity of available materials (Sept et al. 1992). Thus, savanna-dwelling populations of chimpanzees may also be hypothesized to reuse nests at a greater frequency, as these habitats have fewer trees and more extreme seasonality than forests (Hernandez-Aguilar 2009; Lindshield et al. 2017; Wessling et al. 2020). We found that reuse took significantly less time than building a new nest. However, the time saved in reuse is unlikely to represent a biologically significant amount of energy saved and there is currently no evidence that these chimpanzees are more energetically challenged than their forest counterparts (Wessling et al. 2018a, b).

We observed few day nests as data collection focused on night nests of chimpanzees. Bonobos use day nests for rest, for social activities such as grooming and play, and as social "tools" to create and symbolize "personal space" (Fruth and Hohmann 1993). In this study, chimpanzees used nests almost exclusively for rest, although we found feeding remains in and beneath many night nests (F. Stewart pers. obs.), suggesting that individuals either traveled to their nests with food or foraged during the night and returned to their nests with food. We observed no social interactions in night nests. However, the use of day nests for social play, putting infants down, and grooming has been observed in Fongoli (J. Pruetz pers. obs.) and these chimpanzees are not always inactive in their nests overnight when other social activities may occur (Pruetz 2018).

In captivity chimpanzees sleep most of the night on either their right or left side, rather than supine, and spend the least amount of time prone (Videan 2006b). In this study chimpanzees almost exclusively adopted a supine posture immediately after finishing nest construction. Captive orangutans also sleep predominantly on the right or left side, sometimes supine, but rarely prone (Samson and Shumaker 2013). Across the apes a supine posture may to be common on settling (e.g., orangutans: van Schaik 2004), but side-lying may be preferred throughout the night (chimpanzees: Goodall 1962; orangutans: Samson and Shumaker 2013; gorillas: Schaller 1963). Limited overnight observations of chimpanzees sleeping in nests in Mahale suggest that supine sleep may be more disturbed as more movements occurred in supine than side-lying positions during overnight (Zamma and Ihobe 2015). Curling up on one side with the limbs drawn in may help conserve body heat (Goodall 1962), but more research is needed on this topic, especially in the wild. Most primates sleep overnight in a sitting posture, despite often resting during the day in prone or side-lying positions which are constrained by phylogeny and physiology (Matsuda et al. 2017). Sitting upright for overnight sleep may represent a trade-off between selection of peripheral sites on thin branches inaccessible to predators, huddling for thermal regulation, and sleep needs (Matsuda et al. 2017). A supine posture may be more comfortable in an arboreal nest, which tends to have a cup shape, than in captive conditions where great apes make nests from different materials on a flat support structure (Samson and Shumaker 2013; Videan 2006a, 2006b).

That chimpanzees often traveled just before nesting suggests that although chimpanzees' ranging is influenced by distribution of fruit trees (Basabose 2004; Furuichi and Hashimoto 2004), they do not always build nests near feeding trees at Fongoli. Although chimpanzees plan their foraging decisions, they are also highly selective in choosing a nesting site (Janmaat et al. 2014). Chimpanzees are 
highly selective of the tree species and morphology used for nesting, specifically selecting species with more malleable branches, but also taller trees with higher first branches (Baldwin et al. 1981; Fruth and Hohmann 1996; Hernandez-Aguilar et al. 2013; Stanford and O'Malley 2008).

\section{Sex Differences in Nest Building}

Several sex differences in nest building emerged in this study. Females nested higher than males, but not higher within trees as found in bonobos (Fruth 1995). Rather, females selected larger trees than males for building in Fongoli. Females also took longer to build nests.

Female chimpanzees nest higher than males at several study sites (Brownlow et al. 2001; Koops et al. 2006; Pruetz et al. 2008). However, no other studies have reported similar sex differences in time of construction. Bonobo females not only build higher, but also earlier than males (Fruth 1995). We found no such sex differences in the time of initiation of nest building and we found no indication that females initiated nest building more often than males. Because males in both Pan species compete for access to receptive females (Gerloff et al. 1999; Wroblewski et al. 2009), males have been proposed to select locations beneath females to remain close to them and prevent competing males from approaching them (Fruth 1995). This was also proposed as an explanation for ground nesting (Koops et al. 2006). If males mapped their nest positions onto the females', however, we would have expected to see a difference in onset of nesting between males and females in this study.

Higher nesting sites within the canopy may receive more sunlight and so afford a greater volume of thinner twigs, leaves, and leaflets than sites lower in the canopy. However, we could find no studies of the distribution of leaves throughout the canopy in the forests or woodlands of savanna mosaic landscapes, although the density of leaves is greater in the middle canopy of primary rainforest in Gabon (Hladik 1978). In more open vegetation types, there may be no influence of canopy height on leaf density because light penetrates throughout the canopy.

Females may invest more in nest building owing to their having dependent offspring and so having to build larger nests for two occupants for most of their adult lives. Females' nests may therefore need to be more structurally sound and safe to provide a stable platform for infants. Female chimpanzees may also be predisposed to proficiency in material skills such as nest building or tool use. Studies have reported sex differences in termite fishing; for example, female chimpanzees in Gombe termite fished more frequently and ant dipped in longer bouts than did males (McGrew 1979). McGrew also proposed that females show more sophisticated tool using skills, owing to greater nutritional demands from pregnancy and infant care. Alternatively, males may be less proficient because their time spent observing and learning is constrained by time spent maintaining social hierarchy and relationships (Lonsdorf 2005). Our study expands on previous work on bonobo nest building to show that Pan females invest more time in nest building (Fruth 1995), which could result from selection pressure of infant care or a predisposition to learn material skills. Differences in material skills likely emerge during ontogeny. 


\section{Age Differences}

Younger bonobos spend longer building and less time using day nests than older bonobos, even before building night nests independently from their mothers, suggesting that immature infants and juveniles practice nest building first during the day and that their building skills improve and become more efficient over time (Fruth 1995). Our comparisons in this study of nest building by different age and sex classes included only dry season overnight nests and only compared immatures to adult builders, rather than infants, juveniles, or adolescents separately.

The two immature males spent a similar amount of time building to adult males, although this sample size was too small for statistical comparison. Selection of nesting position within trees may be related to body size, as immature males nested higher than males and in more removed locations (as measured by the number of decisions to the nesting spot within the tree) where branches were likely thinner. This may be because immatures are more vulnerable to predation, or alternatively that thinner branches are more easily manipulated by smaller individuals and that immatures can likely be supported by branches of greater compliance without risk of falling, given their smaller size. Longitudinal study of the acquisition of nest building skills in the wild has lagged behind other studies of nest building behavior (Anderson et al. 2019) and is required to better understand the ontogeny of nest building and determine whether sex differences in nest building emerge early in development, as with termite fishing and other behaviors (Lonsdorf 2005; Lonsdorf et al. 2004, 2014).

\section{Conclusions}

Our data are preliminary, because they are only for the dry season and because we observed a small number of individuals and measured a small number of nests per individual. However, the results indicate sex differences in building behavior between males and females, which expands the known sex differences in chimpanzees and other primates (Lonsdorf 2017; Lonsdorf et al. 2014). Further study is needed to investigate how female and male nests differ architecturally, whether females modify their building behavior dependent on the presence of a dependent offspring, and when and how during development these sex differences emerge. Whether the sex differences described in this study result from a disparity in the ability of young males and females to learn skills, as has been proposed for human children and suggested for chimpanzees (see Lonsdorf et al. 2004), remains to be tested thoroughly. We did not examine individual differences, but future studies should examine whether specific building techniques are socially transmitted and vary culturally.

Acknowledgements We are grateful to Johnny Dondo Kante, Mboule Camera, Wali Camara, and Eladjh Saho for their invaluable help and enthusiasm in the field. Special thanks to William McGrew for his enthusiasm, support, guidance, and feedback. We are grateful to Erin Wessling and two anonymous reviewers whose feedback greatly improved this manuscript. Sincere thanks to Alecia Carter whose patient help with statistical issues was invaluable. We are grateful to the Carnegie Trust for Universities of Scotland, Harold Hyam Wingate Foundation, L.S.B. Leakey Foundation, International Primatological Society, and the WennerGren Foundation for Anthropological Research for financial support to FAS and National Geographic Society, Iowa State University, Leakey Foundation, Wenner-Gren Foundation for Anthropological Research, and National Science Foundation for support to JDP. 
Author Contributions FAS conceived, designed, and executed this study; FAS and JDP contributed materials/analysis tools and wrote the manuscript. No other person is entitled to authorship.

Open Access This article is licensed under a Creative Commons Attribution 4.0 International License, which permits use, sharing, adaptation, distribution and reproduction in any medium or format, as long as you give appropriate credit to the original author(s) and the source, provide a link to the Creative Commons licence, and indicate if changes were made. The images or other third party material in this article are included in the article's Creative Commons licence, unless indicated otherwise in a credit line to the material. If material is not included in the article's Creative Commons licence and your intended use is not permitted by statutory regulation or exceeds the permitted use, you will need to obtain permission directly from the copyright holder. To view a copy of this licence, visit http://creativecommons.org/licenses/by/4.0/.

\section{References}

Albert, A., Savini, T., \& Huynen, M. (2011). Sleeping site selection and presleep behavior in wild pigtailed macaques. American Journal of Primatology, 73, 1222-1230.

Anderson, J. R. (2000). Sleep-related behavioral adaptations in free-ranging anthropoid primates. Sleep Medicine Reviews, 4, 355-373.

Anderson, J. R., Ang, M. Y. L., Lock, L. C., \& Weiche, I. (2019). Nesting, sleeping, and nighttime behaviors in wild and captive great apes. Primates, 60(4), 321-332. https://doi.org/10.1007/s10329-019-00723-2.

Baldwin, P. J., Sabater Pi, J., McGrew, W. C., \& Tutin, C. E. G. (1981). Comparisons of nests made by different populations of chimpanzees (Pan troglodytes). Primates, 22, $474-486$.

Barrett, B. J., Monteza-Moreno, C. M., Dogandžić, T., Zwyns, N., Ibáñez, A., \& Crofoot, M. C. (2018). Habitual stone-tool-aided extractive foraging in white-faced capuchins, Cebus capucinus. Royal Society Open Science, 5, 181002. https://doi.org/10.1098/rsos.181002.

Basabose, A. K. (2004). Fruit availability and chimpanzee party size at Kahuzi montane forest, Democratic Republic of Congo. Primates, 45(4), 211-219. https://doi.org/10.1007/s10329-004-0087-7.

Bates, D., Mächler, M., Bolker, B. M., \& Walker, S. C. (2015). Fitting linear mixed-effects models using lme4. Journal of Statistical Software, 67(1). 10.18637/jss.v067.i01

Bernstein, I. S. (1962). Responses to nest building materials of wild born and captive born chimpanzees. Animal Behavior, 10, 1-6.

Biro, D., Sousa, C., \& Matsuzawa, T. (2006). Ontogeny and cultural propagation of tool use by wild chimpanzees at Bossou, Guinea: Case studies in nut cracking and leaf folding. In T. Matsuzawa, M. Tomonaga, \& M. Tanaka (Eds.), Cognitive development in chimpanzees (pp. 476-508). Tokyo: Springer Tokyo. https://doi.org/10.1007/4-431-30248-4_28.

Boesch, C., \& Boesch, H. (1984). Possible causes of sex differences in the use of natural hammers by wild chimpanzees. Journal of Human Evolution, 13(5), 415-440. https://doi.org/10.1016/S0047-2484(84 )80055-X.

Brownlow, A. R., Plumptre, A. J., Reynolds, V., \& Ward, R. (2001). Sources of variation in the nesting behavior of chimpanzees (Pan troglodytes schweinfurthii) in the Budongo Forest, Uganda. American Journal of Primatology, 55(1), 49-55. https://doi.org/10.1002/ajp.1038.

Chapman, C. A. (1989). Spider monkey sleeping sites: Use and availability. American Journal of Primatology, 18, 53-60. https://doi.org/10.1002/ajp.1350180106.

Core Team, R. (2019). R: A Language and Environment for Statistical Computing. Vienna: Austria.

Di Bitetti, M. S., Vidal, E. M. L., Baldovino, M. C., \& Benesovsky, V. (2000). Sleeping site preferences in tufted capuchin monkeys (Cebus apella nigritus). American Journal of Primatology, 50, 257-274.

Emery Thompson, M. E., Muller, M. N., \& Wrangham, R. W. (2012). The energetics of lactation and the return to fecundity in wild chimpanzees. Behavioral Ecology, 23(6), 1234-1241. https://doi.org/10.1093 /beheco/ars 107.

Fruth, B. (1995). Nest and nest groups in wild bonobos (Pan pansicus): Ecological and behavioral correlates. München: Aachen: Shaker: $\mathrm{PhD}$, Ludwig-Maximilians-Universität.

Fruth, B., \& Hohmann, G. (1993). Ecological and behavioral aspects of nest building in wild bonobos (Pan paniscus). Ethology, 94(2), 113-126. https://doi.org/10.1111/j.1439-0310.1993.tb00552.x.

Fruth, B., \& Hohmann, G. (1996). Nest building behavior in the great apes: The great leap forward? In W. C. McGrew, L. F. Marchant, \& T. Nishida (Eds.), Great ape societies (pp. 225-240). Cambridge: Cambridge University Press. 
Furuichi, T., \& Hashimoto, C. (2004). Botanical and topographical factors influencing nesting-site selection by chimpanzees in Kalinzu Forest, Uganda. International Journal of Primatology, 25(4), 755-765. https://doi.org/10.1023/B:IJOP.0000029121.25284.7f.

Gerloff, U., Hartung, B., Fruth, B., Hohmann, G., \& Tautz, D. (1999). Intracommunity relationships, dispersal pattern and paternity success in a wild living community of Bonobos (Pan paniscus) determined from DNA analysis of faecal samples. Proceedings of the Royal Society of London B: Biological Sciences, 266(1424), 1189-1195. https://doi.org/10.1098/rspb.1999.0762.

Goodall, J. M. (1962). Nest building behavior in free-ranging chimpanzee. Annals of the New York Academy of Sciences, 102, 455-467.

Groves, C. P., \& Sabater Pi, J. (1985). From ape's nest to human fix-point. Man, 20(1), 22. https://doi. org/10.2307/2802220.

Gruber, T., Clay, Z., \& Zuberbühler, K. (2010). A comparison of bonobo and chimpanzee tool use: Evidence for a female bias in the Pan lineage. Animal Behavior, 80(6), 1023-1033. https://doi.org/10.1016/j. anbehav.2010.09.005.

Gumert, M. D., Hoong, L. K., \& Malaivijitnond, S. (2011). Sex differences in the stone tool-use behavior of a wild population of Burmese long-tailed macaques (Macaca fascicularis aurea). American Journal of Primatology, 73, 1239-1249.

Hamilton, J. W. (1982). Baboon sleeping site preferences and relationships to primate grouping patterns. American Journal of Primatology, 3, 41-53.

Hernandez-Aguilar, RA. (2009). Chimpanzee nest distribution and site reuse in a dry habitat: implications for early hominin ranging. Journal of Human Evolution, 57(4):350-364. https://oi.org/10.1016/j. jhevol.2009.03.007.

Hernandez-Aguilar, R. A., Moore, J., \& Pickering, T. R. (2007). Savanna chimpanzees use tools to harvest the underground storage organs of plants. Proceedings of the National Academy of Sciences of the USA, 104(49), 19210-19213. https://doi.org/10.1073/pnas.0707929104.

Hernandez-Aguilar, R. A., Moore, J., \& Stanford, C. B. (2013). Chimpanzee nesting patterns in savanna habitat: Environmental influences and preferences. American Journal of Primatology, 75(10), 979-994. https://doi.org/10.1002/ajp.22163.

Hiraiwa-Hasegawa, M. (1989). Sex differences in behavioral development of chimpanzees at Mahale. In P. G. Heltne \& L. A. Marquardt (Eds.), Understanding chimpanzees (pp. 104-115). Cambridge, MA: Harvard University Press.

Hladik, A. (1978). Phenology of leaf production in rain rainforest of Gabon: Distribution and composition of food for folivores. In G. G. Montgomery (Ed.), The ecology of arboreal folivores (pp. 51-71). Washington, DC: Smithsonian Insititution Press.

Janmaat, K. R. L., Polansky, L., Ban, S. D., \& Boesch, C. (2014). Wild chimpanzees plan their breakfast time, type, and location. Proceedings of the National Academy of Sciences of the USA, 111(46), 16343-16348. https://doi.org/10.1073/pnas.1407524111.

Kahlenberg, S. M., \& Wrangham, R. W. (2010). Sex differences in chimpanzees' use of sticks as play objects resemble those of children. Current Biology, 20(24), R1067-R1068. https://doi.org/10.1016/j. cub.2010.11.024.

Koops, K., Humle, T., Sterck, E. H. M., \& Matsuzawa, T. (2006). Ground-nesting by the chimpanzees of the Nimba Mountains, Guinea: Environmentally or socially determined? American Journal of Primatology, 69, 1-13. https://doi.org/10.1002/ajp.

Koops, K., Furuichi, T., Hashimoto, C., \& van Schaik, C. P. (2015). Sex differences in object manipulation in wild immature chimpanzees (Pan troglodytes schweinfurthii) and bonobos (Pan paniscus): Preparation for tool use? PLOS ONE, 10(10), e0139909. https://doi.org/10.1371/journal.pone.0139909.

Krief, S., Cibot, M., Bortolamiol, S., Seguya, A., Krief, J. M., \& Masi, S. (2014). Wild chimpanzees on the edge: Nocturnal activities in croplands. PLoS ONE, 9(10). https://doi.org/10.1371/journal.pone.0109925

Lapuente, J., Arandjelovic, M., Kühl, H., Dieguez, P., Boesch, C., Linsenmair, K.E. (2020). Sustainable peeling of kapok tree (Ceiba pentandra) bark by the chimpanzees (Pan troglodytes verus) of Comoé National Park, Ivory Coast. International Journal of Primatology. https://doi.org/10.1007/s10764-02000152-9. (this issue).

Lindshield, S., Danielson, B. J., Rothman, J. M., \& Pruetz, J. D. (2017). Feeding in fear? How adult male western chimpanzees (Pan troglodytes verus) adjust to predation and savanna habitat pressures. American Journal of Physical Anthropology, 163(3), 480-496. https://doi.org/10.1002/ajpa.23221.

Lodwick, J. L., Borries, C., Pusey, A. E., Goodall, J., \& McGrew, W. C. (2004). From nest to nest: Influence of ecology and reproduction on the active period of adult Gombe chimpanzees. American Journal of Primatology, 64(3), 249-260. https://doi.org/10.1002/ajp.20076. 
Lonsdorf, E. V. (2005). Sex differences in the development of termite-fishing skills in the wild chimpanzees, Pan troglodytes schweinfurthii, of Gombe National Park, Tanzania. Animal Behavior, 70(3), 673-683. https://doi.org/10.1016/j.anbehav.2004.12.014.

Lonsdorf, E. V. (2017). Sex differences in nonhuman primate behavioral development. Journal of Neuroscience Research, 95(1-2), 213-221. https://doi.org/10.1002/jnr.23862.

Lonsdorf, E. V., Eberly, L. E., \& Pusey, A. E. (2004). Sex differences in learning in chimpanzees. Nature, 428(April), 1-2. https://doi.org/10.1038/nature02527.

Lonsdorf, E. V., Markham, A. C., Heintz, M. R., Anderson, K. E., Ciuk, D. J., Goodall, J., \& Murray, C. M. (2014). Sex differences in wild chimpanzee behavior emerge during infancy. PLoS ONE, 9(6), 1-9. https://doi.org/10.1371/journal.pone.0099099.

Matsuda, I., Chapman, C. A., Shi, P., Chua, Y., Mun, S., John, C., \& Clauss, M. (2017). Primate resting postures: Constraints by foregut fermentation? Physiological and Biochemical Zoology, 90(3), 383-391.

McGrew, W. C. (1979). Evolutionary implications of sex differences in chimpanzee predation and tool use. In D. A. Hamburg \& E. R. McCown (Eds.), Perspectives on human evolution (pp. 441-463). Menlo Park, CA: Benjamin/Cummings.

McGrew, W. C. (1992). Chimpanzee material culture: Implications for human evolution. Cambridge: Cambridge University Press.

McGrew, W. C., Baldwin, P. J., \& Tutin, C. E. G. (1981). Chimpanzees in a hot, dry and open habitat: Mr. Assirik, Senegal, West Africa. Journal of Human Evolution, 10, 227-244.

McIntyre, M. H., \& Edwards, C. P. (2009). The early development of gender differences. Annual Review of Anthropology, 38(1), 83-97. https://doi.org/10.1146/annurev-anthro-091908-164338.

Meredith, S. L. (2015). Comparative perspectives on human gender development and evolution. American Journal of Physical Anthropology, 156, 72-97. https://doi.org/10.1002/ajpa.22660.

Moore, J. (1996). Savanna chimpanzees, referential models and the last common ancestor. In W. C. McGrew, L. F. Marchant, \& T. Nishida (Eds.), Great ape societies. Cambridge: Cambridge University Press.

Plumptre, A. J., \& Reynolds, V. (1997). Nesting behavior of chimpanzees: Implications for censuses. International Journal of Primatology, 18, 475-485.

Pruetz, J. D. (2006). Feeding ecology of savanna chimpanzees (Pan troglodytes verus) at Fongoli, Senegal. In G. Hohmann, M. M. Robbins, \& C. Boesch (Eds.), Feeding ecology in apes and other primates: Ecological, physical and behavioral aspects (pp. 161-182). Cambridge: Cambridge University Press.

Pruetz, J. D. (2007). Evidence of cave use by savanna chimpanzees (Pan troglodytes verus ) at Fongoli, Senegal: Implications for thermoregulatory behavior. Primates, 48, 316-319. https://doi.org/10.1007 /s10329-007-0038-1.

Pruetz, J. D. (2018). Nocturnal behavior by a diurnal ape, the West African chimpanzee (Pan troglodytes verus), in a savanna environment at Fongoli, Senegal. American Journal of Physical Anthropology, 166(3), 541-548. https://doi.org/10.1002/ajpa.23434.

Pruetz, J. D., \& Bertolani, P. (2007). Savanna chimpanzees, Pan troglodytes verus, hunt with tools. Current Biology, 17(5), 412-417. https://doi.org/10.1016/j.cub.2006.12.042.

Pruetz, J. D., \& Bertolani, P. (2009). Chimpanzee (Pan troglodytes verus) behavioral responses to stresses associated with living in a savannah-mosaic environment: Implications for hominin adaptations to open habitats. PaleoAnthropology, 2009, 252-262. https://doi.org/10.4207/pa.2009.art33.

Pruetz, J. D., Fulton, S. J., Marchant, L. F., McGrew, W. C., Schiel, M., \& Waller, M. (2008). Arboreal nesting as anti-predator adaptation by savanna chimpanzees (Pan troglodytes verus) in southeastern Senegal. American Journal of Primatology, 70(4), 393-401. https://doi.org/10.1002/ajp.20508.

Pruetz, J. D., Bertolani, P., Boyer-Ontl, K., Lindshield, S., Shelley, M., \& Wessling, E. G. (2015). New evidence on the tool-assisted hunting exhibited by chimpanzees (Pan troglodytes verus) in a savannah habitat at Fongoli, Sénégal. Royal Society Open Science, 2(4). https://doi.org/10.1098/rsos.140507.

Reichard, U. (1998). Sleeping sites, sleeping places, and presleep behavior of gibbons (Hylobates lar). American Journal of Primatology, 46, 35-62.

Samson, D. R., \& Shumaker, R. W. (2013). Documenting orang-utan sleep architecture: Sleeping platform complexity increases sleep quality in captive Pongo. Behavior, 150(8), 845-861. https://doi.org/10.1163 /1568539X-00003082.

Schaller, G. B. (1963). The mountain gorilla: Ecology and behavior. Chicago: University of Chicago Press.

Sept, J., King, B., McGrew, W., Moore, J., Paterson, J., et al (1992). Was there no place like home? A new perspective on early hominid archaeological sites from the mapping of chimpanzee nests [and Comments and Reply]. Current Anthropology, 33(2), 187-207.

Smith, R. L., Hayes, S. E., Smith, P., \& Dickens, J. (2018). Sleeping site preferences in Sapajus cay Illiger 1815 (Primates: Cebidae) in a disturbed fragment of the Upper Paraná Atlantic Forest, Rancho Laguna Blanca, Eastern Paraguay. Primates, 59, 79-88. https://doi.org/10.1007/s10329-017-0626-7. 
Stanford, C. B., \& O’Malley, R. C. (2008). Sleeping tree choice by Bwindi chimpanzees. American Journal of Primatology, 70(7), 642-649. https://doi.org/10.1002/ajp.20539.

Stewart, F. A. (2011). The evolution of shelter: Ecology and ethology of chimpanzee nest building. $\mathrm{PhD}$ disseration, University of Cambridge.

Stewart, F. A., \& Pruetz, J. D. (2013). Do chimpanzee nests serve an anti-predatory function? American Journal of Primatology, 75(6), 593-604. https://doi.org/10.1002/ajp.22138.

Stewart, F. A., Piel, A. K., \& McGrew, W. C. (2011). Living archaeology: Artefacts of specific nest site fidelity in wild chimpanzees. Journal of Human Evolution, 61(4), 388-395. https://doi.org/10.1016/j. jhevol.2011.05.005.

Stewart, F. A., Piel, A. K., Azkarate, J. C., \& Pruetz, J. D. (2018). Savanna chimpanzees adjust sleeping nest architecture in response to local weather conditions. American Journal of Physical Anthropology, 166(3), 549-562. https://doi.org/10.1002/ajpa.23461.

Tagg, N., McCarthy, M., Dieguez, P., Bocksberger, G., Willie, J., et al (2018). Nocturnal activity in wild chimpanzees (Pan troglodytes): Evidence for flexible sleeping patterns and insights into human evolution. American Journal of Physical Anthropology, 166(3), 510-529. https://doi.org/10.1002/ajpa.23478.

Thiry, V., Stark, D. J., Goossens, B., Slachmuylder, J. L., Vercauteren Drubbel, R., \& Vercauteren, M. (2016). Use and selection of sleeping sites by proboscis monkeys, Nasalis larvatus, along the Kinabatangan River, Sabah, Malaysia. Folia Primatologica, 87, 180-196. https://doi.org/10.1159/000449219.

Van Lawick-Goodall, J. (1968). The behavior of free-living chimpanzees in the Gombe Stream Reserve. Animal Behavior Monographs, 1, 161-311. https://doi.org/10.1016/s0066-1856(68)80003-2.

van Schaik, C. P. (2004). Among orangutans: Red apes and the rise of human culture. Cambridge, MA: Belknap Press of Harvard University Press.

Videan, E. N. (2006a). Bed-building in captive chimpanzees (Pan troglodytes): The importance of early rearing. American Journal of Primatology, 68(7), 745-751. https://doi.org/10.1002/ajp.

Videan, E. N. (2006b). Sleep in captive chimpanzee (Pan troglodytes): The effects of individual and environmental factors on sleep duration and quality. Behavioral Brain Research, 169(2), 187-192. https://doi.org/10.1016/j.bbr.2005.12.014.

Wessling, E. G., Deschner, T., Mundry, R., Pruetz, J. D., Wittig, R. M., \& Kühl, H. S. (2018a). Seasonal variation in physiology challenges the notion of chimpanzees (Pan troglodytes verus) as a forest-adapted species. Frontiers in Ecology and Evolution, 6, 60. https://doi.org/10.3389/fevo.2018.00060.

Wessling, E.G., Dieguez, P., Llana, M., Pacheco, L., Pruetz, J.D. Kühl, H.S. (2020). Chimpanzee (Pan troglodytes verus) density and environmental gradients at their biogeographical range edge. International Journal of Primatology. (this issue).

Wessling, E. G., Kühl, H. S., Mundry, R., Deschner, T., \& Pruetz, J. D. (2018b). The costs of living at the edge: Seasonal stress in wild savanna-dwelling chimpanzees. Journal of Human Evolution, 121, 1-11. https://doi.org/10.1016/j.jhevol.2018.03.001.

Wroblewski, E. E., Murray, C. C., Keele, B. F., Schumacher-Stankey, J. C., Hahn, B. H., \& Pusey, A. E. (2009). Male dominance rank and reproductive success in chimpanzees, Pan troglodytes schweinfurthii. Animal Behavior, 77(4), 873-885. https://doi.org/10.1016/j.anbehav.2008.12.014.Male.

Zamma, K. (2014). What makes wild chimpanzees wake up at night? Primates, 55(1), 51-57. https://doi. org/10.1007/s10329-013-0367-1.

Zamma, K., \& Ihobe, H. (2015). Bed making and nocturnal behavior. In K. Hosaka, K. Zamma, M. Nakamura, \& N. Itoh (Eds.), Mahale chimpanzees: 50 years of research (pp. 583-598). Cambridge: Cambridge University Press.

\section{Affiliations}

\section{Fiona A. Stewart ${ }^{1}$ - Jill D. Pruetz ${ }^{2}$}

1 Faculty of Science, School of Natural Sciences and Psychology, Liverpool John Moores University, Liverpool, UK

2 Department of Anthropology, Texas State University, San Marcos, TX, USA 